ture by environment and on heredity. Fo example, we might expect increased webbing of the feet, and this might become hereditary.

Hiram M. Stanley.

LAKE FoREst, ILL., June.

\section{DARKENING OF THE CATHODE IN A} CROOKES TUBE.

A PEAR-SHAPED Crookes tube with a cathode disc in its narrow end has been used extensively by us during the past ten weeks in private experimentation and in public lectures on Röntgen rays. In common with many other experimenters, we have observed that after much usage the glass opposite the cathode disc and the glass about the anode became darkened. But we do not recall having seen any statement recorded regarding the darkening of the cathode disc. When we began using the tube the surface of the aluminium disc was uniformly bright throughout; now there is on the surface facing the broad end of the tube a dark brown ring concentric with the disc. This ring has an internal diameter of about $6 \mathrm{~mm}$., and is darkest near its inner edge, the densest portion being, perhaps, $1 \mathrm{~mm}$. across. Outside of this darkest portion the ring fades off gradually toward the outer edge of the disc. Taken as a whole, the internal and external diameters of the ring are about $5 \mathrm{~mm}$. and $11 \mathrm{~mm}$. respectively. The circular area inside of the dark ring is the brightest part of the disc. The diameter of the disc is about $17 \mathrm{~mm}$.

During the discharge through the tube we now observe what we did not notice before, viz., a pencil of faint bluish light emanating from the circular area of the disc inside the dark ring. The pencil is normal to the disc. The light resembles the blue or purplish light about the anode. The cylindrical pencil is most distinct at the disc and gradually fades away and becomes invisible at a distance from it of about 2 or $3 \mathrm{~cm}$. If, by reversal of the current, the disc is made the anode, then the pencil of blue light cannot be seen, but almost the entire tube is filled with the same purplish light. Sometimes this purplish light fills the tube also when the disc is used as a cathode. In such cases the discharge at the spark gap (placed in series with the tube) is fat and noisy; the tube shows very little fluorescence and the radiation of Röntgen rays is greatly diminished.

FLORIAN CAJORI, William Strieby, Colorado College, Colorado Springs.

\section{SCIENTIFIC LITERATURE.}

Voice Building and Tone Placing, showing a method of relieving injured vocal cords by tone exercises. By H. Holbrook Curtis, Pн. B., M. D. D. Appleton and Company. 1896.

This latest claimant for favor in the difficult field of voice production will be found to contain much that is old to those familiar with the subject of acoustics and some that is as unexpected as it is new. The struggling pupil will find it difficult to extract the pearl of good advice from the shell of lengthy discussion. From the preface one can see that the author realizes at once the difficulty of the problem and what its solution should be, but it is doubtful if he has fulfilled the promise.

The author begins with a brief outline of the history of music, which is followed by a description of the anatomy of the larynx which is naturally all right, until he begins to discuss the operation of the various parts, and here certain discrepancies arise. For example, we are told that there is but one register, or rather that registers are 'fallacies,' and yet in attempting to discuss our control of pitch he refers to reaching a 'stage in the production of the lower register,' where, 'for any other further elevation of pitch, a complete rearrangement of the vocal apparatus is necessary.' Just exactly what the devotees of registers claim. In point of fact, however, if one has the proper use of the voice, the same muscles control the pitch from lowest to highest, without break or interruption.

The above is an example of the uncertainty in which the reader is left; registers are called fallacies, and yet they are discussed at length ; they are assumed to exist and their fundamental differences in mechanism pointed out. Another statement which is very misleading, to say the least, is that air pressure in the lungs affects the pitch of the tone; "the pitch of the tone depends upon the strength of the expiratory pressure." How can we then take a tone 